\title{
Sub-MeV Dark Matter Searches with EDELWEISS: results and prospects
}

\section{H.Lattaud for the Edelweiss collaboration ${ }^{a, *}$}

${ }^{a}$ Univ. Lyon, Univ. Lyon 1, CNRS/IN2P3, IP2I, F-69622, Villeurbanne, France

E-mail: lattaud@ipnl.in2p3.fr

The Edelweiss collaboration performs light Dark Matter (DM) particles searches with germanium bolometers collecting charge and phonon signals. Thanks to the Neganov-Trofimov-Luke effect, a RMS resolution of 0.56 electron-hole pair was obtained on a massive (33.4g) germanium detector operated underground at the Laboratoire Souterrain de Modane. This sensitiviy made possible a search for Dark Photons Down to $1 \mathrm{eV}$ and for DM-electron interactions below $1 \mathrm{MeV} / \mathrm{c}^{2}$. It is the first measurement in cryogenic germanium at such low threshold proving the high relevance of this technology. This is an important step of the development of the Ge detectors with improved performance in the context of the EDELWEISS-SubGeV program.

*** The European Physical Society Conference on High Energy Physics (EPS-HEP2021), ***

*** 26-30 July 2021 ***

*** Online conference, jointly organized by Universität Hamburg and the research center DESY ***

${ }^{*}$ Speaker 


\section{Introduction}

The lack of observation of Weakly Interacting Massive Particle (WIMP) [1] by double-phase detectors [2] and the absence of discovery of beyond the standard model particle at the LHC experiments, has brought back interest in model of light Dark Matter (DM) with masses below the $\mathrm{GeV} / \mathrm{c}^{2}$ scale [3]. Such astroparticles scattering on nuclei could produce nuclear recoils with kinetic energies below $1 \mathrm{keV}$. For light DM particle (masses below $100 \mathrm{MeV} / \mathrm{c}^{2}$ ), most of the kinetic energy is transferred to electrons via collision [4] or absorption [5]. In order to be sensitive to such signal, direct DM search experiments aim to lower their threshold for detecting electronic/nuclear recoils down to few eV. In this context, the EDELWEISS collaboration is adapting its cryogenic germanium detectors with phonon and ionization readout [6]. The EDELWEISS-SubGeV program aims to search for DM particles with masses ranging from the $\mathrm{eV} / \mathrm{c}^{2}$ to $\mathrm{GeV} / \mathrm{c}^{2}$ scale using a $1-\mathrm{kg}$ size array of germanium cryogenic detectors allowing to perform particle identification, installed in its radiopure underground site located in the Laboratoire Souterrain de Modane (LSM). The main limitation to such searches $[5,8,9,16,17]$ is that lowering the detection threshold comes with the cost of particle identification. The EDELWEISS-SubGeV goal is to keep these capabilities by achieving a resolution of $\sigma=10 \mathrm{eV}$ for the phonon channel and $20 \mathrm{eV}_{e e}(\mathrm{eV}$-electron-equivalent) for the ionization channel, while being able to apply biases up to $100 \mathrm{~V}$ for charges collection, in order to exploit the Neganov-Trofimov-Luke (NTL) amplification of the phonon signal [10]. A $1 \mathrm{~kg}$-scale array of germanium cryogenic detectors with such performance, operated in the lowbackground environment of the LSM, could explore sub-GeV DM interaction with nuclear recoils with event-by-event rejection down to a few $10^{-43} \mathrm{~cm}^{2}$, and exploit the NTL boost technique to explore MeV Dark Matter interaction with electrons (down to $10^{-40} \mathrm{~cm}^{2}$ ) and the absorption of $\mathrm{eV}$-scale Dark Photon (down to $k=10^{-15}$ ). The required detector developments, in particular that of a cold HEMT preamplifier for the ionization readout [11], are made in cooperation with the RICOCHET collaboration [12]. Developments related to the improvement of the phonon channel and the operation of detectors at high bias are described in the following.

\section{Recent results}

Toward this development process, the EDELWEISS Collaboration has recently reached a $17.7 \mathrm{eV}$ phonon baseline resolution (RMS) with a $33.4 \mathrm{~g}$ Ge bolometer operated above ground [13]. This detector provided the first sub-GeV constraints on DM-nucleon scattering. In order to achieve sub-electron-hole pair resolution, a detector of the same design was equipped with electrodes to take advantage of the NTL amplification. It was operated at LSM in the ultra-low radioactivity environment of the EDELWEISS-III cryostat [6], in order to perform a search of DM interaction with electrons. The experiment, its results and their interpretation are described in details in Ref. [14]. The detector is a $33.4 \mathrm{~g}$ cylindrical high-purity Ge crystal $(\phi 20 \times 20 \mathrm{~mm})$, with electrodes lithographed on each of the two planar surfaces. The phonon sensor is a Ge neutron-transmutationdoped (NTD), glued on the top of the crystal. The data from the phonon and ionization channels were digitized at a frequency of $100 \mathrm{kHz}$, filtered, averaged, and continuously stored on disk with a rate of 500 samples per second. 

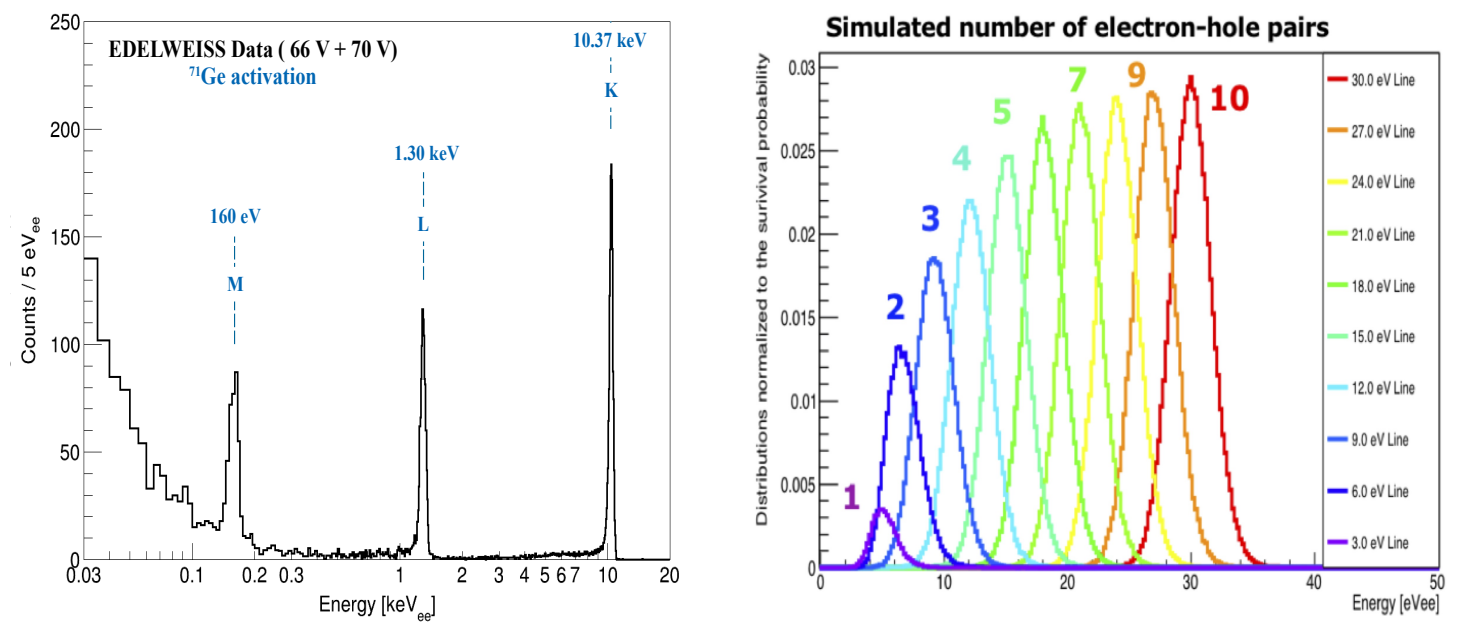

Figure 1: Left: Energy spectrum recorded in the phonon channel with a bias of 66 and $70 \mathrm{~V}$ following the ${ }^{71} \mathrm{Ge}$ activation of the detector. Right: Response of the detector to $N_{\text {pair }}=1$ to 10 electron-hole pairs, obtained by inserting in the data streams appropriately scaled-down ${ }^{71} \mathrm{Ge} K$-line events. These simulated events go through the same triggering, reconstruction and selection procedure used for the real data.

The detectors has been activated using a strong AmBe neutron source. This activation produces the isotope ${ }^{71} \mathrm{Ge}$ which decays with a half-life of 11 days by electron capture in the K, L, M shells, producing X-rays with the total energy of $10.37,1.3$ and $0.16 \mathrm{keV}$. The observed de-excitation lines, are shown on the left panel of in Fig. 1. Thanks to NTL amplification, the $160 \mathrm{eV}$ line is clearly resolved with a width $(\sigma=8 \mathrm{eV})$ limited by the Fano factor. Such lines are absorbed in the bulk of the detector, probing the detector response and allowing a precise low-energy calibration and data efficiency selection characterization. A sample of 858 events from the K-line decay and recorded at the same bias $(78 \mathrm{~V})$ as the DM search data has been used to characterize the efficiency of data processing, triggering and selection as function of the energy. In order to probe the energy dependency, those events were scaled to the desired energy, injected at random time in the data stream and went through the same analysis pipeline as the original data. The right panel of Fig. 1 shows the reconstructed energy distributions of the heat channel for events corresponding to 1 to 10 electron-hole pairs, i.e. 3 to $30 \mathrm{eV}_{e e}$ once the pair creation energy of $3 \mathrm{eV}$ in cryogenic $\mathrm{Ge}$ is considered. This procedure of event simulation account for actual noise condition in data and reconstruction and selection biases as well. The selection cuts are described in detail in Ref. [14]. The efficiency for $3 \mathrm{eV}_{e e}$ events is $4 \%$. It rapidly increases to $22 \%$ at $6 \mathrm{eV}_{e e}$ and stabilize around $60 \%$. The baseline resolution for the DM search data is $1.58 \mathrm{eV}_{e e}$ (RMS), corresponding to 0.53 electron-hole pair.

58 consecutive hours of data has been selected for the search sample. The left panel of Fig. 2 shows the resulting spectrum. A non-blinded data sample of 21 and 9 hours taken just before and after the blinded data sample has been used to tune the data selection cuts and the energy intervals of the search. The total number of events in the selected energy range for the data and the one predicted by the models considered (next paragraph), were used to derive the $90 \%$ CL Limits on the interaction rate of DM particles with electrons. The theoretical distributions of each model as 
a function of the DM particle mass were simulated with the same pulse simulation method used to determine the efficiency. The non-blind sample was used to derive the optimal energy range and the cross-sections that yield a number of count larger than the $90 \%$ C.L. derived from Poisson statistics were excluded. The models considered here are the DM-electron scattering via a heavy or light mediator, corresponding to a form factor $F_{D M}=1$ or $1 / q^{2}$, respectively [4], and the absorption of a Dark Photon via a kinematic mixing $\kappa$ [5]. The resulting limits are shown in Fig. 3. Examples of excluded spectra for two of the models are shown in the left panel of Fig. 2.
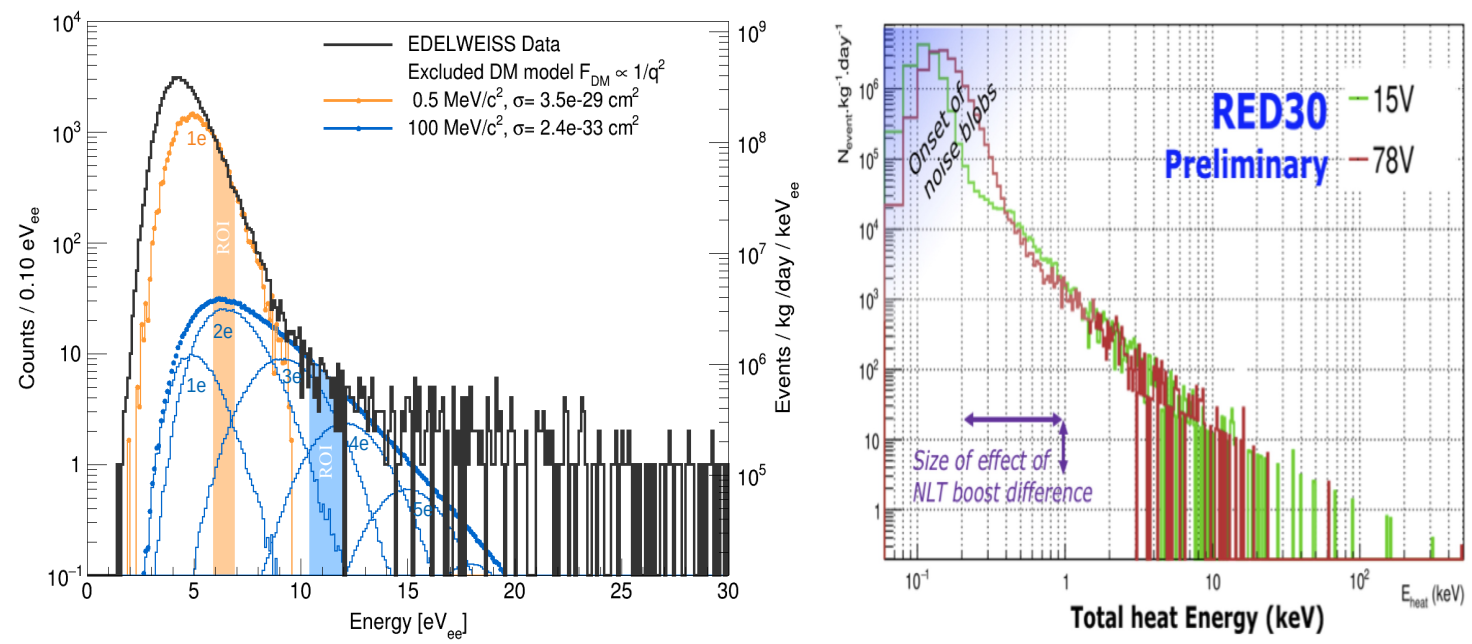

Figure 2: Left: Energy spectrum of the events selected for the DM search (black). The thick blue (orange) histogram is the simulation of the signal excluded at $90 \%$ C.L. for a DM particle with a mass of $10(0.5)$ $\mathrm{MeV} / \mathrm{c}^{2}$, and $F_{D M} \propto 1 / q^{2}$. The corresponding regions of interest (ROI) used to set the upper limits are shown as shaded intervals using the same color code. The thin-line histograms of the same color represent the individual contributions of 1 to 5 electron-hole pairs. Right: Total phonon energy spectra (corresponding to the energy in $\mathrm{keV}_{e e}$ corrected for the NTL boost factor 1+V/3) recorded in the same detector at 15 and $78 \mathrm{~V}$. In both panels, the data are not corrected for efficiencies. Instead, these are taken into account in the simulated signals.

Those DM constrains probe a lower mass range than searches performed by noble gas detectors [16, 17] and are competitive with Si-based detectors searches [7-9].

The better sensitivity of Ge with respect to $\mathrm{Si}$ for a $1 \mathrm{eV} / \mathrm{c}^{2}$ Dark Photon is due to the smaller gap energy in Ge. The achieved sensitivity for Dark Photons with masses below $3 \mathrm{eV} / \mathrm{c}^{2}$ is still very competitive even with the recent results obtained by SENSEI with a Si detector with a very small leakage current. In this mass range the EDELWEISS limit comes from a $90 \%$ C.L. upper bound of $4 \mathrm{~Hz}$ on the efficiency-corrected rate of single-pair events in the detector, which is a contribution to the leakage current of the detector of $<6.4 \times 10^{-19}$ A. Scaling that by the number of target electrons in the detector yield a rate of single-charge events in EDELWEISS that is 25 times larger than the limit achieved in Si by SENSEI [7]. However, for very low masses, this higher backgrounds in Ge is balanced by a more favorable gap energy in Ge. In this context, Ge is a more favorable target for low-mass dark photon searches.

In order to improve the present results, the ongoing work focuses toward lowering the energy 
resolution and understanding the origin of the observed events. The present improvement by an order of magnitude of the detection threshold for electron recoils compared to Ref. [13], in addition to the improved constraints on Dark Matter models helps to understand the origin of the background limiting low-mass searches.

The resolution of the ionization channel $\left(210 \mathrm{eV}_{e e}\right)$ is not sufficient to evaluate if the events shown in the left panel of Fig. 2 are associated with electron-hole pairs creation in the Ge cristal. However, the drift of charges can be checked by comparing the energy spectra recorded at different biases. The total phonon energy $E_{t o t}$ is defined as the sum of the energy of the initial recoiling particle $E_{0}$, plus the contribution of NTL heating due to the work the $N_{\text {pair }}$ drifting, $E_{N T L}=$ $N_{\text {pair }} V$, where $V$ is the absolute value of the bias applied to the detector. The average pair creation energy being $\epsilon_{\gamma}=E_{0} / N_{\text {pair }}$ (with $\epsilon_{\gamma}=3 \mathrm{eV}$ for electron recoils in Ge), for electron recoils and $V$ $=78 \mathrm{~V}$ in our case, the total energy and the initial electron energies relation become:

$$
E_{\text {tot }}=E_{0} \times\left(1+V / \epsilon_{\gamma}\right)=27 \times E_{0}
$$

The right panel of Fig. 2 compares the total heat energy spectra recorded at biases of 15 and 78 V. All contributions from event obviously carrying charges are removed by cutting on ionization signal $E_{\text {ion }}<0$. Below $E_{\text {tot }}=0.4 \mathrm{keV}$, the energy resolution is not good enough to unambiguously separate the read-out noise contribution, as the baseline resolution on $E_{t o t}$ at $78 \mathrm{~V}$ is 10 to $20 \%$ larger than at $15 \mathrm{~V}$. However, the $E_{\text {tot }}$ spectra between 0.4 and $10 \mathrm{keV}$ are very similar in both cases, and are not shifted by the expected 27 factor for electron recoils. Therefore, most of the events above $15 \mathrm{eV}_{e e}$ on the left panel of Fig. 2 are not electron recoils. They correspond to an unidentified population of event that deposit their energy exclusively through phonons, without the creation of electron-hole pairs in the detector.

This type of "Heat-Only" event was reported by EDELWEISS [15], at higher energy though. Improving by an order of magnitude the detection threshold for electron recoils compared to Ref. [13] allowed not only to yield interesting DM constraints, but also provided important information regarding the origin of the background limiting low-mass DM searches.

The next step in the EDELWEISS sub-GeV program is to improve both the phonon resolution of the detectors and the maximum bias at which they can be operated. Developments are ongoing on the study and reduction of the Heat-Only background as it is one of the limiting factor in future searches in EDELWEISS [21]. Systematic studies are under way. For example, a new detector with a novel phonon sensor, consisting in a $100 \mathrm{~nm}$ thick high-impedance NbSi Transition Edge Sensor, lithographed as a $20 \mathrm{~mm}$ diameter spiral on top of a $200 \mathrm{~g}$ Ge crystal. Such sensor eliminates the need of the glue used to fix the NTD sensor. The NbSi film has sensitivity to athermal phonons, this lead to the possibility of evaluating the purely thermal nature of heat-only events. This sensor technique can be combined with NTL amplification. A $200 \mathrm{~g}$ prototype operated at a bias of $66 \mathrm{~V} \mathrm{[20]} \mathrm{already} \mathrm{achieved} \mathrm{a} \mathrm{resolution} \mathrm{of} 5 \mathrm{eV}_{e e}$, and the physics analysis of the data is under way. The collaboration also investigates possible techniques to tag single-charge events in order to reject this background. 

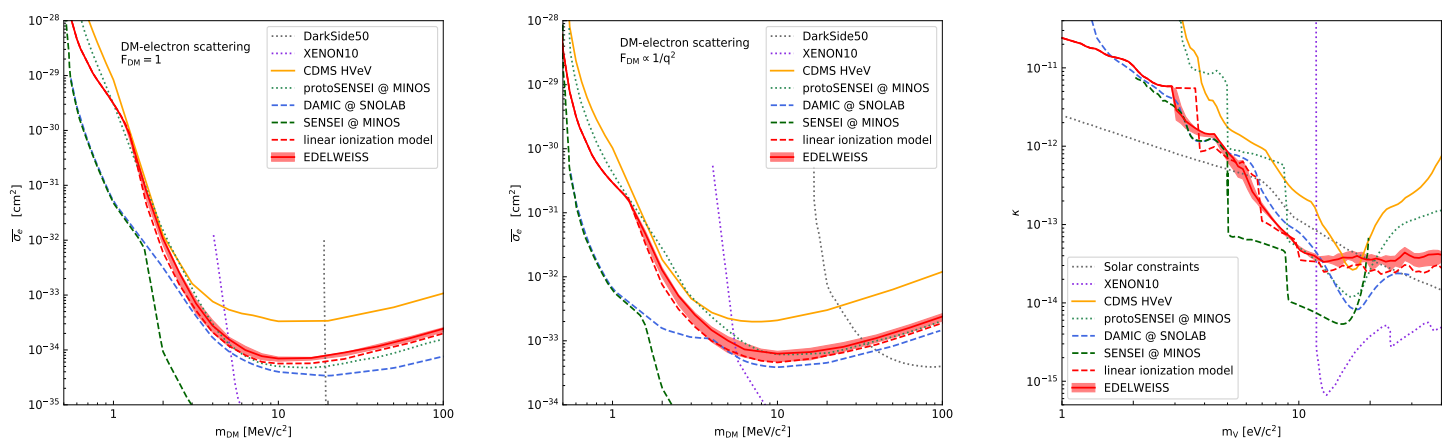

Figure 3: 90\% C.L. upper limit on the cross-section for the scattering of DM particles on electrons, assuming a heavy (left panel) or light (middle panel) mediator. Right panel: 90\% C.L. upper limit on the kinetic mixing $\kappa$ of a Dark Photon. The results from the present work are shown as the red line. The shaded red band and dotted red line represent alternative charge distribution models as described in Ref. [14]. Also shown are constraints from other direct detection experiments [5, 7-9, 16, 17], and solar constraints [18].

\section{Conclusion}

The EDELWEISS-SubGeV program objective is to develop a $1 \mathrm{~kg}$-scale array of germanium cryogenic detectors to be operated at LSM to probe sub-GeV DM interaction with nuclear recoils with event-by-event rejection down to a few $10^{-43} \mathrm{~cm}^{2}$, while keeping the ability to exploit NTL amplification to be able to scan MeV DM interactions with electrons (down to $10^{-40} \mathrm{~cm}^{2}$ ) and the absorption of eV-scale Dark Photon (down to $K=10^{-15}$ ). Recent milestones toward that objective, with interesting physics results, is the achievement of a phonon resolution of $\sigma=17.3 \mathrm{eV}$ on a $33.4 \mathrm{~g}$ detector, and of $\sigma=0.53$ electron-hole pair on a same-size detector biased at 78V. These results yield to competitive DM searches, and in particular confirm the interest of germanium as a low-gap target in searches where the signal is an electron recoil.

The EDELWEISS project is supported in part by the German Helmholtz Alliance for Astroparticle Physics (HAP), by the French Agence Nationale pour la Recherche (ANR) and the LabEx Lyon Institute of Origins (ANR-10-LABX-0066) of the Universite de Lyon within the program "Investissements d'Avenir" (ANR-11-IDEX-00007), by the P2IO LabEx (ANR-10-LABX-0038) in the framework "Investissements d'Avenir" (ANR-11-IDEX-0003-01) managed by the ANR (France), and the Russian Foundation for Basic Research (grant No. 18-02-00159). This project has received funding from the European Union's Horizon 2020 research and innovation programme under the Marie Sktodowska-Curie Grant Agreement No. 838537. 


\section{References}

[1] L. Bergström, Rept. Prog. Phys. 63, 793, (2000), DOI: 10.1088/0034-4885/63/5/2r3;

G. Bertone, D. Hooper, and J. Silk, Phys. Rep. 405, 279, (2005), DOI: 10.1016/j.physrep.2004.08.031.

[2] E. Aprile et al. (XENON Coll.), Phys. Rev. Lett. 121 ,111302, (2018), DOI: 10.1103/PhysRevLett.121.111302; D. S. Akerib et al. (LUX Collaboration), Phys. Rev. Lett. 118, 021303, (2017), DOI: 10.1103/PhysRevLett.118.021303; X. Cui et al. (PandaX-II Coll.), Phys. Rev. Lett. 119, 181302, (2017), DOI: 10.1103/PhysRevLett.119.181302.

[3] M. Battaglieri et al., US Cosmic Visions: New Ideas in Dark Matter 2017: Community Report, arXiv:1707.04591.

[4] R.Essig, M.Fernández-Serra, J.Mardon, A.Soto, T.Volansky, and T.-T. Yu, J. High Energy Phys. 05, 046, (2016), DOI: 10.1007/JHEP05(2016)046.

[5] H. An, M. Pospelov, J. Pradler, and A. Ritz, Phys. Lett. B 747, 331, (2015), DOI: 10.1016/j.physletb.2015.06.018; Y. Hochberg, T. Lin, and K. M. Zurek, Phys. Rev. D 95, 023013 (2017), DOI: 10.1103/PhysRevD.95.023013.

[6] E. Armengaud et al. (EDELWEISS Coll.), JINST 12, P08010, (2017), DOI: 10.1088/17480221/12/08/P08010.

[7] L. Barak et al. (SENSEI Collaboration), Phys. Rev. Lett. 125, 171802, (2020), DOI: 10.1103/PhysRevLett.125.171802.

[8] A. Aguilar-Arevalo et al. (DAMIC Collaboration), Phys. Rev. Lett. 123, 181802, (2019), DOI: 10.1103/PhysRevLett.123.181802.

[9] R. Agnese et al. (CDMS Collaboration), Phys. Rev. Lett. 121, 051301 (2018), DOI: 10.1103/PhysRevLett.121.051301; 122, 069901(E) (2019), DOI: 10.1103/PhysRevLett.122.069901.

[10] P.N. Luke, J. Appl. Phys. 646858 (1988), DOI: 10.1063/1.341976;

B. Neganov and V. Trofimov. Otkryt. Izobret. 146, 215 (1985).

[11] J.B. Filippini et al, High Impedance, low dissipation, and ultra-low noise HEMT-based $1 \mathrm{~K}$ front-end electronics and readout for the CryoCube detector array of the future RICOCHET experiment, J. Low Temp. Phys., this Special Issue (2021); Juillard A et al., J. Low Temp. Phys. 184, 897, (2019), DOI: 10.1007/s10909-019-02269-5.

[12] T. Salagnac et al, (RICOCHET Coll.), Optimization and performance of the CryoCube detector for the future Ricochet low-energy neutrino experiment, J. Low Temp. Phys., LTD19 Special Issue (2021).

[13] E. Armengaud et al. (EDELWEISS Coll.), Phys. Rev. D 99, 082003, (2019), DOI: 10.1103/PhysRevD.99.082003. 
[14] Q. Arnaud et al. (EDELWEISS Coll.), Phys. Rev. Lett. 125, 141301, (2020), DOI: 10.1103/PhysRevLett.125.141301.

[15] L. Hehn et al. et al. (EDELWEISS Coll.), Eur. Phys. J. C 76, 548 (2016), DOI: 10.1140/epjc/s10052-016-4388-y.

[16] I.M. Bloch, R. Essig, K. Tobioka, T.Volansky and T.T. Yu, Journal of High Energy Physics 2017, 87, (2017), DOI: 10.1007/JHEP01(2021)178.

[17] P. Agnes et al. (The DarkSide Collaboration), Phys. Rev. Lett. 121, 111303, (2018), DOI: 10.1103/PhysRevLett.121.111303.

[18] H. An, M. Pospelov, and J. Pradler, Phys. Lett. B 725 , 190, (2013), DOI: 10.1016/j.physletb.2013.07.008; J. Redondo and G. Raffelt, J. Cosmol. Astropart. Phys. 08, 034, (2013), DOI: 10.1088/1475-7516/2013/08/034.

[19] H. Lattaud et al, (EDELWEISS Coll.), Phonon and charge signals from IR and X excitation in the SELENDIS Ge cryogenic detector, J. Low Temp. Phys., LTD19 Special Issue (2021).

[20] S. Marnieros et al, (EDELWEISS Coll.), Ge bolometers with high impedance TES for the EDELWEISS dark matter experiment, J. Low Temp. Phys., LTD19 Special Issue (2021).

[21] Q. Arnaud et al. (EDELWEISS Coll.), Phys. Rev. D 97, 022003, (2018). DOI: 10.1103/PhysRevD.97.022003. 